\title{
(6) OPEN ACCESS \\ Work-focused interventions that promote the labour market transition of young adults with chronic disabling health conditions: a systematic review
}

\author{
Arif Jetha, ${ }_{1}^{1,2}$ Robert Shaw ${ }^{3}$ Adrienne R Sinden, ${ }^{4}$ Quenby Mahood, ${ }^{1}$ \\ Monique AM Gignac, ${ }^{1,2,5}$ Mary Ann McColl, ${ }^{6}$ Kathleen A Martin Ginis ${ }^{3}$
}

'Institute for Work and Health, Toronto, Ontario, Canada ${ }^{2}$ Dalla Lana School of Public Health, University of Toronto, Toronto, Ontario, Canada ${ }^{3}$ School of Health and Exercise Sciences, University of British Columbia, Kelowna, British Columbia, Canada ${ }^{4}$ McMaster University, Hamilton, Ontario, Canada ${ }^{5}$ Krembil Research Institute, Toronto, Ontario, Canada ${ }^{6}$ School of Rehabilitation Therapy, Queen's University, Kingston, Ontario, Canada

Correspondence to

Dr Arif Jetha, Institute for Work and Health, Toronto, ONM5G

2E9, Canada; ajetha@iwh.on.ca

Received 24 August 2018 Revised 3 December 2018 Accepted 17 December 2018 Published Online First 11 January 2019

\section{ABSTRACT}

Objective Young adulthood is an important transitional life phase where careers are established. Young adults with chronic disabling health conditions are underrepresented in the labour market. Our study aims to examine the effectiveness of work-focused interventions that support the labour market transition of young adults with chronic disabling health conditions; and to examine whether the effectiveness of work-focused interventions differ across work transition phase (eg, preparation, entry and sustaining work, employment advancement) and disability type.

Methods A systematic review of articles published between January 1990 and July 2018 was conducted. Medline, EMBASE and PsycInfo were searched, and titles/abstracts and full texts of articles were reviewed for eligibility. Relevant articles were appraised for methodological quality. A best evidence synthesis was applied to medium-quality/high-quality studies to develop recommendations.

Results 5816 articles were identified; 10 articles were relevant and of moderate-high methodological quality. Six intervention categories were identified which focused on young adults with mental health or intellectual/learning disabilities $(n=3)$ and addressed employment preparation $(n=10)$ and/or work entry $(n=9)$. No interventions addressed at-work issues or career advancement. Strong evidence existed for tailored supported employment (SE) interventions having a positive impact on preparation and entry into competitive employment. Also, moderate evidence existed for the positive impact of SE on preparation and entry into competitive employment for young adults with mental health conditions.

Conclusions Tailored SE is recommended to foster preparation and entry into the labour market. Evidencebased interventions are needed to facilitate sustained work and career advancement of young adults living with different disabling health conditions.

\section{Check for updates}

(C) Author(s) (or their employer(s)) 2019. Re-use permitted under CC BY-NC. No commercial re-use. See rights and permissions. Published by BMJ.

To cite: Jetha $A$

Shaw $R$, Sinden $A R$, et al.

Occup Environ Med

2019:76:189-198.

\section{INTRODUCTION}

Young adults living with chronic and disabling health conditions are under-represented within the labour market. ${ }^{1}$ Of concern, challenges at the early career stage can have a long-term impact on involvement in paid work and affect the ability to access resources (eg, income, nutritious food and safe housing) that provide pathways to better health. ${ }^{2}{ }^{3}$ Interventions that support the employment participation of young adults living with chronic disabling health conditions can advance

\section{Key messages}

What is already known about this subject?

- The young adult life phase is characterised by vocational transitions that mark entry into and advancement within the labour market.

- Young adults with chronic disabling health conditions face barriers to finding and sustaining productive employment.

- It is unclear what work-focused interventions would be beneficial to young adults with different chronic disabling health conditions as they transition into the labour market.

What are the new findings?

- Strong evidence existed for tailored supported employment interventions having a positive impact on competitive employment outcomes.

- Moderate evidence existed for tailored supported employment interventions having a positive impact on competitive employment outcomes for young adults with mental health conditions.

- Few other evidence-based interventions exist that address the transitional employment needs of young adults with disabling health conditions.

How might this impact on policy or clinical practice in the foreseeable future?

- To facilitate the transition into work of young adults with disabling conditions, tailored supported employment interventions should be implemented.

- Additional research is required to examine interventions that facilitate sustained work and career advancement

health and quality of life. Our review synthesised evidence regarding work-focused interventions that promote the employment of young adults living with chronic disabling health conditions.

Young adulthood, spanning 18-35 years, is characterised by several transitions (ie, discrete vocational changes) that mark entry into the labour market including exiting educational roles, preparation and entry into paid work and advancement within one's job. ${ }^{45}$ Research indicates that difficulties with employment in young adulthood can have a 'scarring effect' and contribute to adverse work (eg, unemployment, missed work days, earning less 
pay) and health outcomes (eg, psychological distress) that can extend across adulthood. ${ }^{67}$ The current generation of young adults (often referred to as millennials) face unique challenges with employment. When compared with previous generations, millennial young adults possess higher levels of formal education and technological literacy, but are less likely to be employed in full-time permanent jobs and more likely to face income insecurity. ${ }^{8} 9$ It is within these challenging socioeconomic conditions that young people with disabilities are entering the labour market.

Despite the existence of legislation which protects against workplace discrimination and mandates reasonable job accommodation, ${ }^{10} 11$ young adults with chronic and disabling health conditions are only half as likely to participate in employment than their peers without a disability. ${ }^{12}{ }^{13}$ Data from industrialised countries like Canada and the USA indicate that young adults with disabling health conditions are more likely to report underemployment, precarious working conditions and at-work productivity losses. ${ }^{1} 14$ Also, young adults with disabilities have a lower median yearly income compared with their peers without a disability and are more likely to rely on income supports. ${ }^{15} 16$ Income and employment inequity between those with and without a disabling health condition widens with older age. $^{15}$

Interventions that are work-focused (ie, where the specific intention is to promote employment engagement) play an important role in addressing the physical and psychosocial workplace barriers experienced by people with disabling health conditions. ${ }^{18}{ }^{18}$ However, little evidence currently exists which can guide the development of policies and programmes that enhance the employment of young adults with chronic disabling health conditions. Systematic reviews of studies of working-aged samples $(\approx 18-65$ years $)$ with different disabling health conditions indicate the importance of multidimensional work-focused interventions that include workplace modification, health and rehabilitation care, supported job placement and work-related training. ${ }^{17-22}$ Another recent review of studies of vocational programmes of youth (15-25 years) with physical disabilities found that workplace-based training, job-specific mentorship and family engagement were intervention components that had the potential to improve participation in paid and unpaid work roles. ${ }^{23}$ Of note, the methodological quality of the intervention studies in this review was not reported. Hence, it is unclear what work-focused interventions would be beneficial to young adults with different disabling health conditions as they transition into the labour market.

Using a rigorous systematic review methodology, our study examines the following research questions: (1) What work-focused interventions are most effective in supporting the employment of young adults with chronic disabling health conditions? (2) Does the effectiveness of work-focused interventions differ for young adults living with different chronic disabling health conditions (eg, mental health, intellectual/learning, physical and speech/hearing/visual disabilities)? (3) Do interventions and their effectiveness differ across the period of transition into the labour market (eg, preparation, entry, sustaining work and advancement within employment)?

\section{METHODS}

We used a systematic review methodology designed to synthesise evidence in the field of occupational health and safety and aimed at generating recommendations for practice. ${ }^{17}$ Investigative team members had experience with the systematic review process and specific backgrounds in information sciences, epidemiology, social sciences, rehabilitation and health sciences, occupational health and public policy. Embedded within the review process was engagement with relevant stakeholders including young adults with disabilities, employment support practitioners and public policy experts. ${ }^{24}$ The review protocols, described below, were registered with PROSPERO (CRD42018104550) and align with Preferred Reporting Items for Systematic Reviews and Meta-Analyses guidelines.

\section{Question development}

At the outset, the investigative team and stakeholders participated in a series of consultations where research questions were generated. During consultations, stakeholders expressed a lack of evidence regarding the most effective interventions that could be used to facilitate preparation, entry and advancement within the labour market of young adults with disabilities. Stakeholders also described an absence of evidence regarding whether interventions should differ based on disability type. In collaboration with stakeholders, the study team also decided to examine work-focused interventions within Organisation for Economic Co-operation and Development (OECD) countries which have similar socioeconomic contexts.

\section{Literature search}

Search terms were developed iteratively with input from the lead author, information scientist, review team members and stakeholders. All database-specific search terms are available in online supplement 1. Collaboratively, search terms were refined to follow a PICO framework and capture the population of young adults with chronic disabling health conditions, work-focused interventions, comparison groups and work outcomes (table 1). To address our research questions, we searched for any disabling chronic health condition that could affect young adults including mental health (eg, depression), intellectual/learning (eg, attention deficit disorder), physical (eg, juvenile arthritis) or speech/hearing/visual disability. Database-specific controlled vocabulary terms and keywords were included. The terms within each category were combined using a Boolean OR operator and terms across the four main categories were combined using a Boolean AND operator. Medline (OVID), EMBASE (OVID) and PsycInfo (OVID) were searched for articles published between January 1990 and September 2017 to identify work-focused interventions for current and previous generations of young adults with chronic disabling health conditions. The search was subsequently updated in July 2018. Aligning with previous occupational health and safety reviews, research prior to 1990 was

\begin{tabular}{|c|c|}
\hline PICO category & Description \\
\hline Population & $\begin{array}{l}\text { Young adults with chronic disabling health conditions } \\
\text { Young adults living with any health condition that is chronic and } \\
\text { results in prolonged or episodic disability. We included conditions } \\
\text { that can be categorised as resulting in mental health, intellectual/ } \\
\text { learning, physical, or speech/hearing/visual disability. }\end{array}$ \\
\hline Intervention & $\begin{array}{l}\text { Work-focused intervention } \\
\text { Intervention designed to explicitly impact work participation. }\end{array}$ \\
\hline Comparison & Any comparator group \\
\hline Outcomes & $\begin{array}{l}\text { Work participation } \\
\text { Any measure of labour market activity. }\end{array}$ \\
\hline
\end{tabular}

Specific search terms directly align with the PICO framework, and are presented in online supplement 1 . 
considered informative but excluded to account for advancements with workplace, policy and health systems levels that have occurred in OECD countries. ${ }^{18}$ Non-English studies are indexed in the three databases with English-transcribed titles and abstracts. Accordingly, we captured non-English references using English search terms. Search terms were customised to align with each database's specific controlled vocabulary. Reference lists of included studies were also examined to identify references not found in the literature search. The search yields were combined in a citation manager software. Once duplicates were removed, titles and abstracts were imported into Microsoft Excel to facilitate the screening processes.

\section{Relevance screen}

Articles were included if they were primary research, published $\geq$ 1990, focused on a work-specific intervention, within an OECD country, ${ }^{25}$ and where the sample of interest was young adults (18-35 years) with any chronic disabling health condition. We included studies when the sample's mean age fell between 18 and 35 years, and age range was $<45$ years or $>16$ years. Articles which had a broader age range were only included when sample characteristics and intervention effects were reported for young adults. Our operationalisation of young adulthood aligns with theoretical research on the young adult life phase and enabled us to capture various definitions used in the literature. ${ }^{520}$ Intervention studies could be randomised or non-randomised designs but had to have a comparator or control group. ${ }^{17}$ All languages were included in our search. Exclusion criteria comprised (1) non-intervention studies (eg, observational research); (2) secondary research (eg, systematic reviews); (3) non-work-focused interventions (eg, clinical interventions where employment was not an intended outcome); (4) commentary/editorial or case studies; and (5) studies where no statistical intervention effect was recorded. While previously published systematic reviews or meta-analysis studies were not eligible, their references were checked to identify relevant articles.

Titles and abstracts of references identified in the search were divided among three reviewers for relevance screening, such that each reference was screened by two reviewers independently. A quality control step was implemented to ensure inter-rater reliability and limit bias; 5\% of titles and abstracts were examined by two reviewers and findings were compared. Reviewers came to consensus on any disagreements and consulted the third reviewer in cases that could not be resolved. Articles that met the first level of screening were carried forward for a full-text review. Two independent reviewers applied the same inclusion and exclusion criteria to examine each article. Disagreements between the two reviewers were discussed in team meetings. Moderate-high inter-rater reliability was identified in title/abstract and full-text reviews, suggesting that reviewers were consistently applying inclusion/exclusion criteria to the screening processes.

\section{Quality appraisal and data extraction}

Each relevant article was appraised for methodological quality by two independent reviewers. A 25 -item quality assessment tool was used to assess study methodological (ie, study design and objectives, level of recruitment, intervention characteristics and intensity, outcomes and analysis) ${ }^{18}$ (online supplement 2). The appraisal tool has been used in previous occupational health and safety systematic reviews and provided an evaluation of internal, external and statistical validity of each article. ${ }^{26}$ During team meetings, reviewers were required to reach consensus on the application of the quality assessment tool for each study. Once consensus was reached, methodological quality appraisal scores were assigned to each study based on a weighted sum score of the quality criteria. Weightings were created through a consensus building exercise where members of the research team and study stakeholders ranked quality appraisal criterion based on level of importance with regards to methodological dimensions that were most important to addressing our research questions $(1=$ somewhat important; 3 =very important). Weightings are reported in online supplement 2 . Using the weightings, a final quality score was generated and converted to a percentage. Studies were ranked as high $(\geq 85 \%)$, medium $(50 \%-84 \%)$ or low quality $(<50 \%) .{ }^{27}$ While informative, low-quality studies were ultimately excluded from the review because of their potential risk of bias and methodological limitations. Data from included studies were extracted to create summary tables which included sample description, intervention details and work outcomes. Interventions that used similar mechanisms and conceptual principles were grouped together.

\section{Evidence synthesis}

Evidence synthesis considered the quality, quantity and consistency of evidence to draw practice-based conclusions for each intervention category. Given that studies varied in their length of observation, design and confounding variables, pooled effect estimates were not calculated. However, a best evidence synthesis approach was taken to generate messages for policy or practice based on the level of evidence available. ${ }^{28}$

The effectiveness of an intervention was determined using criteria that were applied to each study. In particular, a study exhibiting a positive effect was characterised by findings which showed a significantly positive result and either no negative results or no null effects. A negative effect intervention referred to a study exhibiting any negative effect. Intervention effects, quality ratings and number of studies were all considered to determine the level of evidence for each category of intervention uncovered in the review. Evidence was synthesised using an algorithm that considers the quality and quantity of studies and consistency of study findings ${ }^{29}$ (table 2).

\begin{tabular}{|c|c|c|c|c|}
\hline Level of evidence & Minimum quality & Minimum quantity & Consistency & Strength of message \\
\hline Strong & $\mathrm{High}^{*}(\mathrm{H})$ & 3 & $3 \mathrm{H}$ agree; if $3^{+}$studies, $\geq 3 / 4$ of the $\mathrm{M}$ and $\mathrm{H}$ agree & Recommendations \\
\hline Moderate & Mediumt (M) & $2 \mathrm{H}$ or $2 \mathrm{M}$ and $1 \mathrm{H}$ & $2 \mathrm{H}$ agree or $2 \mathrm{M}$ and $1 \mathrm{H}$ agree; if $3^{+}$studies, $\geq 2 / 3$ of the $\mathrm{M}$ and $\mathrm{H}$ agree & Practice considerations \\
\hline Limited & & $1 \mathrm{H}$ or $2 \mathrm{M}$ or $1 \mathrm{M}$ and $1 \mathrm{H}$ & $2(\mathrm{M}$ and/or $\mathrm{H})$ agree; if 2 studies, $>1 / 2$ of the $\mathrm{M}$ and $\mathrm{H}$ agree & Not enough evidence to \\
\hline Mixed & & 2 & Findings are contradictory & $\begin{array}{l}\text { make recommendations or } \\
\text { practice considerations }\end{array}$ \\
\hline Insufficient & \multicolumn{4}{|c|}{ Medium quality studies that do not meet above criteria } \\
\hline
\end{tabular}




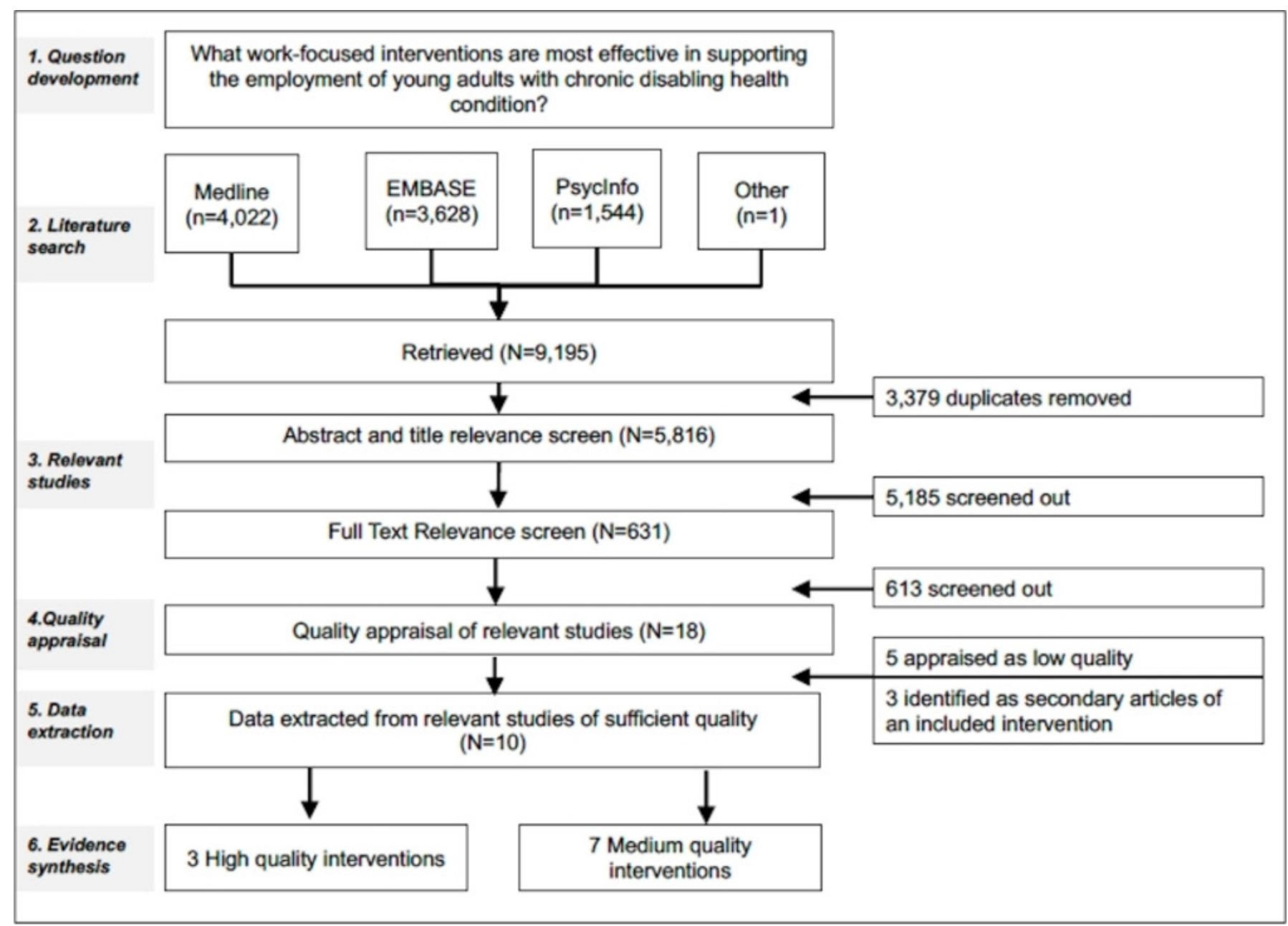

Figure 1 Flow chart of study identification, selection and synthesis.

The algorithm has been used in several systematic reviews in the field of occupational health and safety ${ }^{18}{ }^{30}$ to guide policy and practice recommendations. An intervention that is supported by a strong level of evidence contributes to specific recommendations for policy and practice. An intervention supported by a moderate level of evidence contributes to practice considerations. In contrast, limited, mixed or insufficient evidence levels contribute to a lack of evidence to guide policies or practices. Interventions that were supported by moderate to strong evidence levels were examined further to determine if their effect differed based on disability type or phase of work transition. Practice recommendations were generated in collaboration with study stakeholders to develop specific messages that could be disseminated to knowledge users.

\section{RESULTS}

\section{Literature search and relevancy screen}

Spanning January 1990-July 2018, our search yielded 5816 articles from various databases and after removing duplicates. Following title/abstract relevancy screening, 5185 articles were excluded. Most common reasons for exclusion were studies not focusing on young adults (45\%) and/or a specific work intervention (40\%). Full-text reviews of the remaining 631 manuscripts resulted in 18 studies being carried forward for quality appraisal. Ten articles of moderate to high quality were identified as relevant for data extraction (figure 1). For three work-focused interventions, we identified two published articles. Multiple articles describing the same intervention were grouped together in table 1, but only the primary article was included in the evidence synthesis. All eligible articles were in English language.

\section{Quality appraisal}

Using the quality appraisal tool, three studies were high quality ( $\geq 85 \%$ of quality appraisal score) and seven articles were of medium quality $(50 \%-84 \%$ of quality appraisal score). Five articles were appraised rated as being of low quality $(<50 \%$ of quality appraisal score) and were excluded from data extraction and evidence synthesis. Consistent across all medium-quality and high-quality studies, reasons for receiving a lower quality appraisal score were non-random selection of the study sample $(n=10)$ and potential for co-intervention $(n=10)$. Intervention articles that were appraised as low quality tended to not adequately describe sample inclusion/exclusion criteria $(n=5)$, baseline sample characteristics $(n=3)$, sample attrition $(n=4)$ or key intervention mechanisms $(n=3)$. Low-quality articles were also characterised by suboptimal statistical analyses $(n=5)$ and did not control for important confounding variables in their analysis $(n=5)$.

\section{Data extraction}

Of the 10 studies, 5 were randomized trials, 2 were non-randomised trials and 3 used other study designs (eg, cohort studies, post-test evaluation) (table 3). Over half of the intervention 


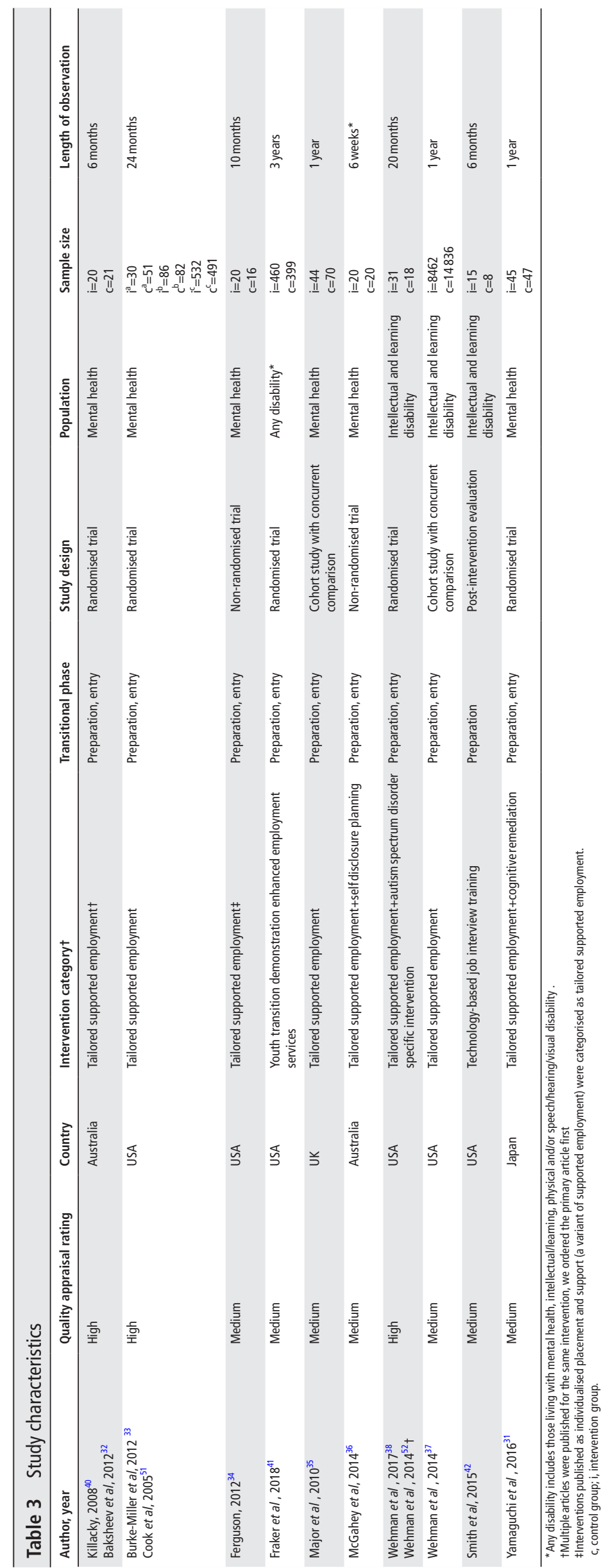


studies were conducted in the USA $(n=6)$. The remaining were conducted in Australia $(n=2)$, the UK $(n=1)$ and Japan $(n=1)$. Across the studies, over half had an observation length of $\geq 1$ year and ranged from 6 weeks to 3 years.

\section{Interventions}

Five main intervention categories were uncovered in our systematic review that support the employment of young adults with disabling health conditions (table 3 ).

\section{Tailored supported employment (SE) $(n=8)^{31-38}$}

$\mathrm{SE}$ is a job training programme where a person with a disabling health condition is integrated within a business to acquire competitive employment. SE participants obtain tailored vocational coaching in a number of areas including interpersonal skills, behavioural self-monitoring, problem solving, requesting assistance, transportation, and workplace policies and procedures. The approach is collaborative and involves a multidisciplinary support team (eg, vocational rehabilitation service providers, healthcare professionals, families, educational agencies and employers). Notably, SE includes disability-awareness training within the workplace in which the participant is placed. Individualised placement and support are considered a variant of SE that is applied to people with mental health conditions. Individualised placement and support interventions were grouped under the SE intervention category. ${ }^{39}$

\section{Tailored SE in combination with a disease-specific work intervention} $\left(\mathrm{SE}^{+}\right)(\mathrm{n}=2)^{31} 3840$

Several interventions combined SE with disability-specific work interventions. For instance, one study implemented autism-specific interventions including behavioural analysis, support/ consultation from an autism specialist and workplace autism awareness. ${ }^{38}$ The second offered cognitive remediation to people with mental illness including training on sustaining attention, psychomotor speed, building learning memory capacity and managing cognitive problems. ${ }^{31}$

Tailored SE in combination with self-disclosure training $(n=1)$

One intervention offered an SE intervention that was combined with training on self-disclosure. Participants in this intervention were asked to identify which items of personal and health-related information they wish to share with others and were coached on the development of a strategy for disclosure. ${ }^{36}$

\section{Youth transition demonstration (YTD) enhanced employment services $(n=1)$}

YTD provided a cluster of employment services including individualised work-based experiences, empowerment building, family support and connection to service providers (eg, healthcare providers, education programme, transportation, assistive technologies). At the policy level, YTD participants also received waivers for income support that enabled them to engage in the labour market without loss of social security. ${ }^{41}$

\section{Technology-based job interview training $(n=1)$}

A virtual reality-based training programme was provided that simulated a job interview with a virtual organisational human resource representative. The job interview training programme provided customisable interview questions and simulated a rapport with an interviewer that would mimic a real-life job interview. Participants were provided with a score and specific feedback to improve interview skills. ${ }^{42}$
All 10 interventions focused on preparation for employment and 9 interventions also targeted entry into work. No intervention focused on sustaining employment or career advancement. Six interventions were administered to young adults living with mental health conditions (eg, psychosis, major depressive disorder), ${ }^{31-36}$ three were administered to young adults living with intellectual and learning disability (eg, autism spectrum disorder $)^{373842}$ and one focused broadly on young adults with different disabilities. ${ }^{41}$

\section{Work outcomes}

Eight studies examined the impact of the intervention on competitive employment, which is defined as meaningful integrated employment that is consistent with a person's career interests and skills, and where wages are at the market rate. ${ }^{39}$ Competitive employment was measured dichotomously (competitively employed; not competitively employed). Three studies also assessed employment in any job including part-time or full-time paid work in contract or permanent positions (employed; not employed). Few studies also collected information on job tenure (ie, days employed for pay), employment income (ie, paid weekly earnings) and hours worked (ie, weekly hours worked) (table 4).

\section{Evidence synthesis}

Evidence was synthesised for each intervention category and specific practice-based messages were generated (table 5). A strong level of evidence existed for SE on competitive employment (three high $(\mathrm{H})$ and four medium (M) quality studies). Practice-based recommendations can be drawn from this level of evidence; implementing an SE programme is recommended for young adults with disabilities to prepare for and secure competitive employment. Moderate evidence was available for the effect of SE on employment in any job $(2 \mathrm{H}$ and $1 \mathrm{M})$. Practice-based considerations can be drawn from this level of evidence; SE can be considered to promote employment in any job. Findings showed insufficient evidence for the effect of SE on other work outcomes (eg, income, hours worked or job tenure). Also, insufficient evidence was identified for the use of other interventions including $\mathrm{SE}^{+}, \mathrm{SE}$ and self-disclosure training, YTD and technology-based job interview training.

When examining whether interventions differed for young adults with different chronic disabling health conditions, a moderate level of evidence existed for the use of SE to support competitive employment for young adults with mental health conditions $(2 \mathrm{H}$ and $3 \mathrm{M})$. Thus, SE should be considered as an effective intervention to increase the likelihood of competitive employment for young adults with mental health conditions. There was not enough evidence to support the use of SE for competitive employment of young adults living with other disabling health conditions. There was also insufficient evidence to examine whether the interventions would be beneficial for other phases of the transition to employment.

\section{DISCUSSION}

Young adults with chronic disabling health conditions face challenges finding and sustaining paid work and may benefit from specialised support. Our systematic review is one of the first to synthesise evidence regarding the effectiveness of work-focused interventions for young adults with chronic disabling health conditions. Interventions that support the transition into the labour market address a critical social determinant of health and provide young adults with pathways to better health and quality of life. Only a handful of work-focused interventions 


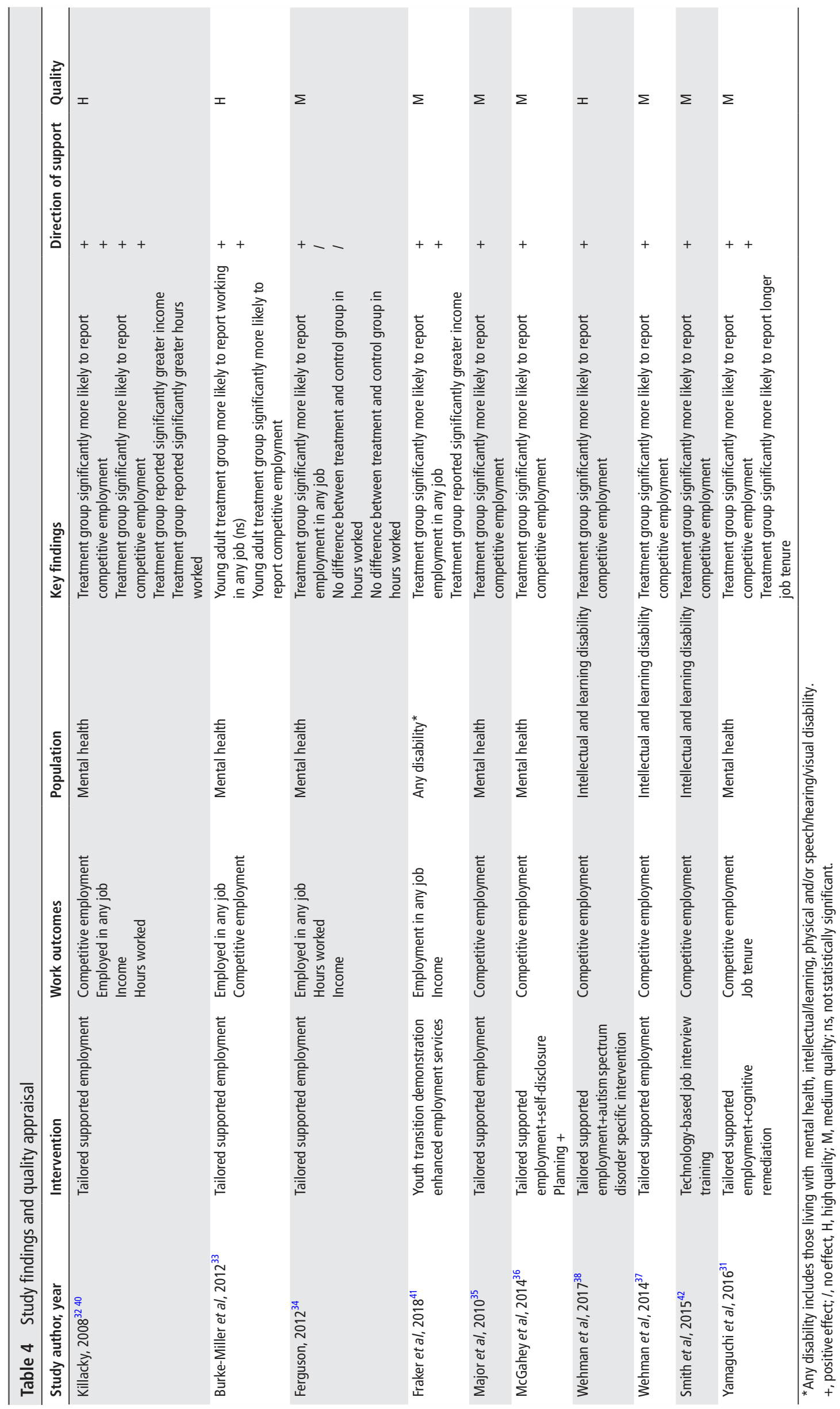


Table 5 Level of evidence for work-focused interventions targeting young adults and accompanying messages

\begin{tabular}{|c|c|c|c|}
\hline Levels of evidence & $\begin{array}{l}\text { Intervention, number of high }(\mathrm{H}) \text { and medium } \\
\text { (M) quality studies }\end{array}$ & Work outcome & Message* \\
\hline \multicolumn{4}{|c|}{ What work-focused policies or programmes are most effective in supporting the employment transition of young adults with chronic disabling health condition? } \\
\hline Strong (positive) & Tailored supported employment (3H, 4M) & Competitive employment & $\begin{array}{l}\text { Implementing tailoredsupported employment } \\
\text { is recommended to help young adults with } \\
\text { chronic disabling health conditions prepare and secure } \\
\text { competitive employment. }\end{array}$ \\
\hline Moderate (positive) & Tailored supported employment (2H, 1M) & Employment in any job & $\begin{array}{l}\text { Implementing a supported employment should be } \\
\text { considered to help young adults with chronic disabling } \\
\text { health conditions prepare and secure employment in } \\
\text { any job }\end{array}$ \\
\hline Limited (positive) & Tailored supported employment (2M) & Hours worked & $\begin{array}{l}\text { Not enough evidence from the scientific literature to } \\
\text { guide current policies/practices }\end{array}$ \\
\hline Limited (positive) & Tailored supported employment (2M) & Income & $\begin{array}{l}\text { Not enough evidence from the scientific literature to } \\
\text { guide current policies/practices }\end{array}$ \\
\hline \multirow[t]{7}{*}{ Insufficient } & $\begin{array}{l}\text { Tailored supported employment+self disclosure } \\
\text { planning }(1 \mathrm{M})\end{array}$ & Competitive employment & $\begin{array}{l}\text { Not enough evidence from the scientific literature to } \\
\text { guide current policies/practices }\end{array}$ \\
\hline & $\begin{array}{l}\text { Tailored supported employment+autism spectrum } \\
\text { disorder specific intervention (1M) }\end{array}$ & Competitive employment & \\
\hline & $\begin{array}{l}\text { Tailored supported } \\
\text { employment+cognitive remediation (1M) }\end{array}$ & Competitive employment & \\
\hline & $\begin{array}{l}\text { Tailored supported } \\
\text { employment+cognitive remediation ( } 1 \mathrm{M})\end{array}$ & Job tenure & \\
\hline & $\begin{array}{l}\text { Youth transition demonstration enhanced } \\
\text { employment services }(1 \mathrm{M})\end{array}$ & Employment in any job & \\
\hline & $\begin{array}{l}\text { Youth transition demonstration enhanced } \\
\text { employment services }(1 \mathrm{M})\end{array}$ & Income & \\
\hline & Technology-based job interview training (1M) & Competitive employment & \\
\hline
\end{tabular}

Does the effectiveness of work-focused policies and programmes differ for young adults living with different chronic disabling health conditions? Mental health

Moderate (positive) Tailored supported employment $(2 \mathrm{H}, 3 \mathrm{M}) \quad$ Competitive employment

Implementing a supported employment programme should be considered to help young people with mental health conditions secure competitive employment

Intellectual and learning

Limited (positive) Tailored supported employment $(1 \mathrm{H}, 1 \mathrm{M}) \quad$ Competitive employment

Not enough evidence from the scientific literature to guide current policies/practices

Do interventions and their effectiveness differ across the period of transition into the labour market? Insufficient

*A majority of work-focused interventions identified in the systematic review were applied to young adults with mental health and intellectual/learning disabilities.

Recommendations should be interpreted accordingly.

$\mathrm{H}$, high-quality study; M, medium-quality study.

were uncovered through our systematic review. Among those that were found, sufficient evidence only existed for SE as an effective intervention that can help young adults with disabilities prepare and find competitive employment. Our systematic review underscores the need for additional development and evaluation of interventions that would support young adults with disabling conditions as they enter the workforce and advance within their careers.

A main finding from our study was the limited number of medium-quality to high-quality intervention studies which addressed the employment needs of young adults with chronic disabling health conditions. Only 10 intervention studies were identified from our systematic review, a majority of which were based in the USA. Most intervention studies focused on preparation and entry into employment. Outcome measures examined in the intervention studies tended to focus on whether or not a participant was employed competitively or in any job. No studies examined at-work outcome measures (eg, absenteeism or presenteeism) or career growth (eg, job promotion, changes in income or seniority or perceived quality of employment ${ }^{43}$ ). Findings suggest that there is minimal high-quality evidence to guide the development of approaches for the long-term employment of young adults with chronic disabling health conditions. There is a need to further develop and evaluate interventions that address the unique transitional work experiences of young adults with disabling health conditions using randomised trials of representative community-based samples.

Notably, sufficient evidence existed to recommend the use of SE to facilitate preparation and entry into competitive employment for young adults with chronic disabling health conditions. SE interventions identified in our review were multidimensional and included several common features such as competitive employment placements, job coaching, collaboration of a multidisciplinary research team and changing workplace attitudes towards employees with disabilities. It may be that the cluster of SE services are effective in addressing the physical and psychosocial barriers that young adults with disabling health conditions face at the early career stage. ${ }^{20}$ Of the SE intervention studies uncovered in this review, observational lengths ranged from 6 weeks to 2 years. It is unclear if SE can be helpful for young adults to sustain employment. Studies are required to expand on findings by examining the effect of SE on longer-term work 
experiences. Additionally, studies are required within a broader range of OECD countries to further investigate the effectiveness of SE in different contexts. Interestingly, several interventions included SE in combination with a disability-specific intervention $\left(\mathrm{SE}^{+}\right) .{ }^{31}{ }^{38}$ Although there was not sufficient evidence from our systematic review to support $\mathrm{SE}^{+}$, tailoring work-focused interventions to a particular disability has the potential to enable a young person to better navigate specific employment challenges they may encounter. Research is needed to examine the efficacy of $\mathrm{SE}^{+}$interventions on the employment of young adults with different disabling conditions.

Studies consistently indicated that mental health disorders are one of the most significant causes of work disability in industrialised countries. ${ }^{44-47}$ Our systematic review provided moderate evidence for the use of SE for the competitive employment of young adults with mental health conditions. Results align with a previous meta-analysis, which also highlights the benefits of SE for the employment of working-aged adults with mental health conditions. ${ }^{48}$ A hallmark of SE interventions is work placement combined with health care and vocational rehabilitation support delivered by a multidisciplinary team. ${ }^{49}$ Accordingly, SE may offer the specific skills for young adults to balance their work with the management of mental health symptoms. ${ }^{49}$ With the exception of one study, no other intervention studies identified in our review focused on physical (eg, juvenile arthritis, multiple sclerosis) or speech/hearing/visual disabilities, or traumatic injuries (eg, spinal cord injury, traumatic brain injury). There is a paucity of interventions that address the employment needs of young adults living with some of the most commonly reported chronic disabling health conditions, many of which can be associated with significant challenges with involvement in paid work. ${ }^{44} 47$

Lastly, only one intervention identified in our review specifically addressed policy-level conditions to support the employment of young adult with disabling conditions. ${ }^{41}$ Some studies indicate that policy-level factors such as the loss of disability benefits represent a commonly reported disincentive to entering the labour market for people with a disability. Interestingly, Farkar et al offered social assistance waivers to young adult study participants so that they could participate in a job placement and training programme without loss of income support. ${ }^{41}$ Participants who received the combined intervention were more likely to hold paid employment and report greater income compared with the control group. Additional research is required to expand on the role of policy-level interventions in influencing the employment participation of young adults living with different chronic disabling health conditions.

Strengths of this systematic review included the utilisation of a rigorous methodology that has been designed and applied within the field of occupational health and safety, and involve stakeholder engagement and the use of a comprehensive methodological quality appraisal tool to evaluate risk of bias and methodological limitations. To answer our research questions, we included studies that reported statistical intervention effects, and excluded grey and qualitative literature. ${ }^{50}$ Additional research is required to synthesise other forms of evidence to enhance our understanding of work-focused interventions for young adults with disabilities, and to determine the contexts in which interventions are most effective. Even though we used a comprehensive search strategy that drew from the expertise of an information scientist and study stakeholders, it is possible that searching through additional databases could have yielded relevant studies that may have been missed. Creating weighted quality appraisal scores through a consensus-based approach enabled the research team to grade the methodological rigour of each study in terms of its ability to answer our research questions. At the same time, this process could potentially result in certain studies being excluded. Lastly, due to heterogeneity in intervention components and study designs, we did not perform a meta-analysis. Instead, we conducted a best evidence synthesis to determine levels of evidence and to provide practitioners with overarching recommendations. ${ }^{28}$

For young adults with chronic disabling health conditions, the transition into the labour market plays an important role in determining working experiences across the life course. Our systematic review of work-focused interventions suggests that young adults with disabling health conditions may benefit from SE. It is recommended that practitioners implement $\mathrm{SE}$ as an intervention to support preparation and entry into competitive employment. At the same time, findings also suggest an overarching absence of interventions of high methodological quality that address the needs of young adults with disabling health conditions as they sustain employment or advance within their career. Also, limited interventions existed for young people with physical or speech/hearing/visual disabilities. In conclusion, there is a need to further develop interventions which support the employment of young adults with chronic disabling health conditions. Enhancing our understanding of work-focused interventions will provide an evidence base that can inform strategic policy and programmatic design, and offer tailored approaches for income generation and health promotion.

Acknowledgements The authors would like to acknowledge the support of Emma Irvin, Dwayne Van Eerd and Kimberly Cullen at the Institute for Work and Health for their support implementing the systematic review methodology. We are grateful for the support of Joanna Liu for her assistance with the literature search and article retrieval. We would also like to thank all of the study stakeholders who contributed to the systematic review process.

Contributors AJ, RS, MG, MAMG and KAMG were involved in study conceptualisation, methodological design and stakeholder engagement. AJ, RS, QM, MG, MAMG and KMG developed systematic review search terms. AJ, RS, AS, QM performed title/abstract and full-text reviews. AJ, RS and AS conducted appraisals of methodological quality. All authors contributed to manuscript development.

Funding This work was supported by a Partnership Grant from the Social Sciences and Humanities Research Council of Canada (\#895-2013-1021) for the Canadian Disability Participation Project.

Competing interests None declared.

Patient consent for publication Not required.

Provenance and peer review Not commissioned; externally peer reviewed.

Open access This is an open access article distributed in accordance with the Creative Commons Attribution Non Commercial (CC BY-NC 4.0) license, which permits others to distribute, remix, adapt, build upon this work non-commercially, and license their derivative works on different terms, provided the original work is properly cited, appropriate credit is given, any changes made indicated, and the use is non-commercial. See: http://creativecommons.org/licenses/by-nc/4.0/.

\section{REFERENCES}

1 World Health Organization. In: Hartley S, Ilagan V, Rosamond M, eds. World health report on disability et al. Switzerland: GenevaWorld Health Organization and World Bank, 2011.

2 Ahonen EQ, Fujishiro K, Cunningham T, et al. Work as an inclusive part of population health inequities research and prevention. Am J Public Health 2018;108:306-11.

3 Marmot M, Friel S, Bell R, et al. Closing the gap in a generation: health equity through action on the social determinants of health. The Lancet 2008;372:1661-9.

4 Ryan P. The School-to-Work Transition: A Cross-National Perspective. J Econ Lit 2001;39:34-92.

5 Arnett JJ. Emerging adulthood: the winding road from late teens through the twenties. New York, NY: Oxford University Press, 2004.

6 Scarpetta S, Sonnet A, Manfredi T. Rising Youth Unemployment During The Crisis: How to Prevent Negative Long-term Consequences on a Generation? OfEC-oa D, OECD Social, employment and migration working papers. edn. Paris, France, 2010. 
7 Helbling LA, Sacchi S. Scarring effects of early unemployment among young workers with vocational credentials in Switzerland. Empirical Research in Vocational Education and Training 2014;6:12.

8 De Hauw S, De Vos A. Millennials' career perspective and psychological contract expectations: does the recession lead to lowered expectations? J Bus Psychol 2010;25:293-302.

9 Levenson AR. Millennials and the world of work: an economist's perspective. J Bus Psychol 2010;25:257-64.

10 U.S. Department of Justice CRDPAS. The Americans with Disabilities Act : Title /I technical assistance manual : covering state and local government programs and services. Washington, D.C.: U.S. Dept. of Justice, Civil Rights Division, Public Access Section, 2005.

11 Ontario Human Rights Commission. Human rights at work. 3rd Edn, 2008.

12 Canada S. A profile of persons with disabilities among Canadians aged 15 years or older, 2012. Ottawa, ON, 2017.

13 U.S. Department of Labor Bureau of Labor Statistics. College enrollement and work activity of high school graduates. Secondary College enrollement and work activity of high school graduates. 2018 www.bls.gov/news/news.release/hsgc.nr0.htm

14 Jetha A, Badley E, Beaton D, et al. Unpacking early work experiences of young adults with rheumatic disease: an examination of absenteeism, job disruptions, and productivity loss. Arthritis Care Res 2015;67:1246-54.

15 Turcotte M. Persons with disabilities and employment: Statistics Canada, 2014

16 Kaplan GA, Pamuk ER, Lynch JW, et al. Inequality in income and mortality in the United States: analysis of mortality and potential pathways. BMJ 1996;312:999-1003.

17 Furlan AD, Gnam WH, Carnide N, et al. Systematic review of intervention practices for depression in the workplace. J Occup Rehabil 2012;22:312-21.

18 Cullen KL, Irvin E, Collie A, et al. Effectiveness of workplace interventions in return-towork for musculoskeletal, pain-related and mental health conditions: an update of the evidence and messages for practitioners. J Occup Rehabil 2018;28:1-15.

19 Guzmán J, Esmail R, Karjalainen K, et al. Multidisciplinary rehabilitation for chronic low back pain: systematic review. BMJ 2001;322:1511-6.

20 Jetha A, Gignac MAM, Bowring J, et al. Supporting arthritis and employment across the life course: a qualitative study. Arthritis Care Res 2018;70:1461-8.

21 Padkapayeva K, Posen A, Yazdani A, et al. Workplace accommodations for persons with physical disabilities: evidence synthesis of the peer-reviewed literature. Disabil Rehabil 2017;39:2134-47.

22 Crowther RE, Marshall M, Bond GR, et al. Helping people with severe mental illness to obtain work: systematic review. BMJ 2001;322:204-8.

23 Hanif S, Peters $\mathrm{H}, \mathrm{McD}$ ougall $\mathrm{C}$, et al. A systematic review of vocational interventions for youth with physical disabilities. factors in studying employment for persons with disability: how the picture can change: Emerald Publishing Limited, 2017:181-202.

24 Keown K, Van Eerd D, Irvin E. Stakeholder engagement opportunities in systematic reviews: knowledge transfer for policy and practice. J Contin Educ Health Prof 2008;28:67-72.

25 OECD. About the OECD. Secondary About the OECD. 2018 http://www.oecd.org/ about/

26 Cook TD, Campbell DT, Shadish W. Experimental and quasi-experimental designs for generalized causal inference: Houghton Mifflin Boston, 2002.

27 Kennedy CA, Amick BC, Dennerlein JT, et al. Systematic review of the role of occupational health and safety interventions in the prevention of upper extremity musculoskeletal symptoms, signs, disorders, injuries, claims and lost time. J Occup Rehabil 2010;20:127-62.

28 Slavin RE. Best-Evidence synthesis: an alternative to meta-analytic and traditional reviews. Educational Researcher 1986;15:5-11.

29 Institute for Work and Health. Evidence and quality saying what works clearly!. Toronto: Institute for Work and Health, 2007.

30 Van Eerd D, Munhall C, Irvin E, et al. Effectiveness of workplace interventions in the prevention of upper extremity musculoskeletal disorders and symptoms: an update of the evidence. Occup Environ Med 2016;73:62-70.
31 Yamaguchi S, Sato S, Horio N, et al. Cost-effectiveness of cognitive remediation and supported employment for people with mental illness: a randomized controlled trial. Psychol Med 2017;47:53-65.

32 Baksheev GN, Allott K, Jackson HJ, et al. Predictors of vocational recovery among young people with first-episode psychosis: findings from a randomized controlled trial. Psychiatr Rehabil J 2012;35:421-7.

33 Burke-Miller J, Razzano LA, Grey DD, et al. Supported employment outcomes for transition age youth and young adults. Psychiatr Rehabil J 2012;35:171-9.

34 Child and youth care forum. Adapting the individual placement and support model with homeless young adults. Springer: Child and youth care forum, 2012.

35 Major BS, Hinton MF, Flint A, et al. Evidence of the effectiveness of a specialist vocational intervention following first episode psychosis: a naturalistic prospective cohort study. Soc Psychiatry Psychiatr Epidemiol 2010;45:1-8.

36 McGahey E, Waghorn G, Lloyd C, et al. Formal plan for self-disclosure enhances supported employment outcomes among young people with severe mental illness. Early Interv Psychiatry 2016;10:178-85.

37 Wehman P, Chan F, Ditchman N, et al. Effect of supported employment on vocational rehabilitation outcomes of transition-age youth with intellectual and developmental disabilities: a case control study. Intellect Dev Disabil 2014;52:296-310.

38 Wehman P, Schall CM, McDonough J, et al. Effects of an employer-based intervention on employment outcomes for youth with significant support needs due to autism. Autism 2017;21:276-90.

39 Drake RE, Bond GR. The future of supported employment for people with severe mental illness. Psychiatr Rehabil J 2008;31:367-76.

40 Killackey E, Jackson HJ, McGorry PD. Vocational intervention in first-episode psychosis: individual placement and support v. treatment as usual. Br J Psychiatry 2008:193:114-20.

41 Fraker TM, Crane KT, Honeycutt TC, et al. The youth transition demonstration project in Miami, Florida: Design, implementation, and three-year impacts. J Vocat Rehabil 2018;48:79-91.

42 Smith MJ, Fleming MF, Wright MA, et al. Brief report: vocational outcomes for young adults with autism spectrum disorders at six months after virtual reality job interview training. J Autism Dev Disord 2015;45:3364-9.

43 Martin Ginis KA, Evans MB, Mortenson WB, et al. Broadening the conceptualization of participation of persons with physical disabilities: a configurative review and recommendations. Arch Phys Med Rehabil 2017;98:395-402.

44 Jetha A, Chen C, Mustard C, et al. Longitudinal examination of temporality in the association between chronic disease diagnosis and changes in work status and hours worked. Occup Environ Med 2017;74:184-91.

45 Dooley D. Unemployment, underemployment, and mental health: conceptualizing employment status as a continuum. Am J Community Psychol 2003;32():9-20.

46 Veldman K, Reijneveld SA, Ortiz JA, et al. Mental health trajectories from childhood to young adulthood affect the educational and employment status of young adults: results from the TRAILS study. J Epidemiol Community Health 2015;69:588-93.

47 Goetzel RZ, Long SR, Ozminkowski RJ, et al. Health, absence, disability, and presenteeism cost estimates of certain physical and mental health conditions affecting U.S. employers. J Occup Environ Med 2004;46:398-412.

48 Campbell K, Bond GR, Drake RE. Who benefits from supported employment: a metaanalytic study. Schizophr Bull 2011;37:370-80.

49 Bond GR, Drake RE. Making the case for IPS supported employment. Adm Policy Ment Health 2014;41:69-73.

50 Mahood Q, Van Eerd D, Irvin E. Searching for grey literature for systematic reviews: challenges and benefits. Res Synth Methods 2014;5:221-34.

51 Cook JA, Leff HS, Blyler CR, et al. Results of a multisite randomized trial of supported employment interventions for individuals with severe mental illness. Arch Gen Psychiatry 2005;62:505-12.

52 Wehman PH, Schall CM, McDonough J, et al. Competitive employment for youth with autism spectrum disorders: early results from a randomized clinical trial. J Autism Dev Disord 2014;44:487-500. 